\title{
Validação de um sistema de previsão para a queima das pontas das folhas da cebola
}

\author{
Leandro Luiz Marcuzzo
}

Instituto Federal Catarinense - IFC/Campus Rio do Sul, CP 441, CEP 89163-356, Rio do Sul-SC, Brasil. Autor para correspondência: Leandro Luiz Marcuzzo (leandro.marcuzzo@ifc.edu.br) Data de chegada: 26/06/2019. Aceito para publicação em: 14/10/2019.

$10.1590 / 0100-5405 / 225651$

\section{RESUMO}

Marcuzzo, L.L. Validação de um sistema de previsão para a queima das pontas das folhas da cebola. Summa Phytopathologica, v.46, n.1, p.4145, 2020.

Com o objetivo de validar um sistema de previsão, com diferentes níveis de severidade, comparados à pulverização convencional no controle da queima das pontas da cebola, foram conduzidos experimentos em Rio do Sul/SC durante os ciclos de cultivo de 2017, 2018 e 2019. Os programas de pulverização foram estabelecidos de acordo com valores diários de severidade estimada (SE) do sistema de Marcuzzo \& Haveroth atribuindose valores acumulados de 20,25 , e $30 \mathrm{SE}$ e no sistema convencional com pulverizações a cada 5 e 7 dias, comparado com a testemunha não tratada com fungicidas. Com exceção da testemunha, não houve diferença significativa entre os tratamentos quanto à produtividade em todos os ciclos. A AACPD, a severidade final e a taxa de progresso da doença não diferiram entre os tratamentos, mas no sistema de previsão com SE 30 o número de pulverizações foi de 40; 33 e 25\% menor em relação ao sistema de aplicação semanal nos três anos de avaliação.

Palavras-chave: Allium cepa, previsão de doenças, epidemiologia, Botrytis squamosa.

\section{ABSTRACT}

Marcuzzo, L.L. Validation of a forecast system for Botrytis leaf blight of onion. Summa Phytopathologica, v.46, n.1, p.41-45, 2020.

\begin{abstract}
Aiming to validate a forecast system with different severity levels, compared to conventional spraying to control Botrytis leaf blight of onion, experiments were conducted in Rio do Sul, Santa Catarina State (SC), Brazil, during the crop seasons in 2017, 2018 and 2019. The spraying programs were established according to the daily values of estimated severity (ES) obtained by the system of Marcuzzo \& Haveroth, assigning accumulated values of 20, 25 and $30 \mathrm{ES}$, and the conventional system with spraying at
\end{abstract}

every 5 and 7 days, compared to the control not treated with fungicides. Except for control, there was no significant difference among treatments for productivity in all cycles. The area under the disease progress curve (AUDPC), the final severity and the disease progress rate did not differ among treatments, but the forecast system with ES of 30 allowed a number of spraying 40, 33 and 25\% lower than the weekly application system in the three years of evaluation.

Keywords: Allium cepa, plant disease forecaster, epidemiology, Botrytis squamosa.

A cultura da cebola (Allium cepa L.) ocupa o terceiro lugar entre as hortaliças de maior expressão econômica do Brasil, sendo de grande relevância para pequenos produtores da região sul. Segundo dados do IBGE (9), a cultura da cebola ocupou 51.967 hectares no Brasil em 2018, com uma produção de 1.622 .106 toneladas e um rendimento médio de $31.220 \mathrm{Kg}_{\text {ha }}{ }^{-1}$. O Estado de Santa Catarina apresenta a maior área de cultivo de cebola, produzindo 431.759 toneladas na safra 2018, o que apresenta $25 \%$ do total produzido do país, com mais de $70 \%$ concentrando-se na região do Alto Vale do Itajaí (5).

A queima das pontas causada por Botrytis squamosa (Walker) é uma doença de grande importância no alto vale do Itajaí, já que a época de cultivo coincide com condições de temperaturas amenas $\left(\leq 22^{\circ} \mathrm{C}\right)$ e alta umidade $(\geq 90 \%)$. A doença incide principalmente na fase de muda, resultando em mais de $50 \%$ de redução do estande de mudas para transplante, podendo também, indiretamente, afetar o desenvolvimento normal do bulbo, devido ao menor número de folhas sobreviventes na planta (27).

Muitas das doenças de plantas têm sido controladas por métodos empíricos com consequente uso desnecessário de agrotóxicos e aumento dos custos de produção, comprometendo a rentabilidade financeira da cultura, além de impactar o meio ambiente e a possibilidade de resíduos no produto chegar à mesa do consumidor (3).

O desenvolvimento de uma doença depende da interação entre ambiente, patógeno e hospedeiro. Quanto ao ambiente, a temperatura e o molhamento foliar são fundamentais para o processo epidemiológico, podendo ser determinados através de modelos matemáticos $(10,22)$.

Mediante esse contexto, o melhor manejo de controle inclui a previsão de doenças, em que estas são correlacionadas com a variação do ambiente, principalmente durante o processo da infecção (18). Segundo Bergamim Filho \& Amorim (4), os sistemas de previsão de doenças de plantas são representações simplificadas da realidade e preveem o início ou o desenvolvimento futuro de uma doença (2, $12,17)$, além da previsão do momento adequado à pulverização com agrotóxicos (16).

Para a queima das pontas das folhas da cebola existe o sistema de previsão Botcast (18) desenvolvido nas condições do Canadá e o Blight-alert e seus complementos nos Estados Unidos da América (24, $25,26)$, porém são sistemas complexos e pouco funcionais no sistema produtivo, devido às diversas variáveis que devem ser inseridas para seu funcionamento. Sistemas simplificados como o de Marcuzzo 
\& Haveroth (14) são de simples interpretação, pois são avaliadas a temperatura e as horas de molhamento foliar, necessitando para seu uso saber apenas qual o valor de severidade estimada acumulada para realizar a pulverização e se realmente apresentará viabilidade o manejo da doença.

Mediante ao exposto, esse trabalho teve como objetivo validar um sistema de previsão para a queima das pontas das folhas da cebola causado por B. squamosa.

\section{MATERIAL E MÉTODOS}

A validação do sistema de previsão da queima das pontas das folhas da cebola foi conduzida no Instituto Federal Catarinense - IFC/Campus Rio do Sul, no município de Rio do Sul - SC, (Latitude: 27¹1'07" S e Longitude: 49³9'39' W, altitude 655 metros acima do nível do mar), durante os períodos de 17 de abril a 30 de junho de 2017; 16 de abril a 29 de junho de 2018 e 16 de abril a 28 de junho de 2019 , totalizando 11 semanas após a semeadura.

Os dados meteorológicos foram obtidos de uma estação Davis ${ }^{\circledR}$ Vantage Vue 300m localizada ao lado do experimento. Os dados médios obtidos durante a condução do experimento foram 15,$8 ; 15,1$ e $16,9^{\circ} \mathrm{C}$ para temperatura do ar; $18 ; 13,1$ e 16,1 horas de umidade relativa do ar $\geq 90 \%$ e $485 ; 171$ e $243 \mathrm{~mm}$ de precipitação pluvial acumulada em 2017, 2018 e 2019 respectivamente.

Três gramas de sementes de cebola cultivar Empasc 352/Bola Precoce foram semeadas no canteiro em experimento constituído de blocos casualizados com cinco tratamentos e quatro repetições. Cada repetição apresentava uma área de 1,00 x 1,00 m, totalizando no mínimo 600 plantas por repetição. Para avaliação da doença, dez plantas em cada parcela foram previamente escolhidas e demarcadas aleatoriamente. A calagem, a adubação, e os tratos culturais seguiram as normas da cultura (15), não se sendo utilizado inseticidas devido a não ocorrência de insetos no período de avaliação.

A testemunha absoluta, sem pulverização, apenas para comprovação da doença na área constou de tratamento nas mesmas condições, ficando distante a 10 metros dos demais tratamentos.

Para que houvesse inóculo do patógeno na área, mudas de cebola com 30 dias de idade foram inoculadas com auxílio de um atomizador portátil com uma suspensão $\left(\right.$ de $\left.10^{4}\right)$ conídios de B. squamosa, sendo transplantadas após 24 horas de câmara úmida a cada um metro linear ao redor do experimento, no dia da semeadura. Também foram depositados cinco escleródios do patógeno produzidos pela técnica de Marcuzzo et al. (13) entre as parcelas, para também servir de inóculo primário da doença na área.

Para o controle da queima das pontas foram comparados os seguintes regimes de pulverização com mancozeb $(80 \%)+$ oxicloreto de cobre $(50 \%)$ na dose de $250 \mathrm{~g}+200 \mathrm{~g} \mathrm{pc} \cdot \mathrm{hl}^{-1}$, baseado no modelo descrito por Marcuzzo \& Haveroth (14) expresso em SE = $0,008192 *\left(\left((x-5)^{1,01089}\right) *\left((30-x)^{1,19052}\right)\right) *(0,33859 /(1+3,77989 * \exp$ $(-0,10923 * y)))$, onde SE, representa o valor da severidade estimada $(0,1) ; x$, a temperatura $\left({ }^{\circ} \mathrm{C}\right)$ e $y$, o molhamento foliar (horas) que foi substituído pela umidade relativa $\geq 90 \%$.

Atribuiu-se os tratamentos com valores acumulados de SE de 0,20; 0,25 , e 0,30 comparados com sistema convencional com pulverização a cada 5 e 7 dias. A pulverização no sistema de previsão foi realizada quando o somatório diário dos valores de $\operatorname{SE}(020 ; 0,25 ; 0,30)$ foi atingido, sendo então zerado o somatório e iniciada nova contagem dos valores de severidade diários. Adicionalmente, a cada ocorrência de $25 \mathrm{~mm}$ de chuva, todos os tratamentos eram pulverizados, zerados e reiniciava-se a contagem do somatório dos valores de severidade.

As pulverizações nos sistemas de previsão e convencional iniciaram-se 15 dias após a semeadura, no estádio fenológico de chicote, feitas com pulverizador costal eletrônico Jetbras ${ }^{\circledR}$ calibrado para 400 L.ha ${ }^{-1}$.

A severidade da doença foi avaliada em função da porcentagem visual de cada folha infectada (6) na planta a intervalos regulares de sete dias. A severidade da doença ao longo do ciclo foi integralizada e calculada a área abaixo da curva de progresso da doença (AACPD), através da fórmula: AACPD $=\sum[(y 1+y 2) / 2] *(t 2-t 1)$, onde $y 1$ e $y 2$ referem-se a duas avaliações sucessivas da intensidade da doença realizadas nos tempos $t 1$ e $t 2$ ( 7 dias), respectivamente.

Nas dez plantas avaliadas para a severidade da doença determinouse a produtividade da biomassa fresca $(\mathrm{g})$ total de cada muda.

As médias da AACPD, da severidade final, da biomassa fresca de muda (g) e a taxa de infecção aparente proposta por Vanderplank (23) entre os regimes de pulverização, foram submetidas à análise de variância pelo teste de $\mathrm{F}$ e quando significativas foram comparadas pelo teste de Tukey 5\%. A testemunha foi submetida à análise estatística pelo teste de Dunnett 5\% para verificar o efeito da testemunha com os tratamentos.

\section{RESULTADOS E DISCUSSÃO}

Respectivamente nos anos de 2017, 2018 e 2019, os sistemas previsão resultaram em 6,7 e $8(\mathrm{SE}=20) ; 5,6$ e $5(\mathrm{SE}=25) ; 3$, 4 e 3 $(\mathrm{SE}=30)$ pulverizações, comparadas às $6,10 \mathrm{e} 9$ prescritas pelo sistema de pulverização fixa a cada 5 dias; e 5, 6 e 4 pulverizações a cada 7 dias (Tabela 1). Pode-se constatar que o sistema $\mathrm{SE}=0,30$ resultou no menor número de pulverizações em todos os anos de avaliação, enquanto o sistema $\mathrm{SE}=0,20$ proporcionou um aumento de 20 até $50 \%$ no número de pulverizações nos anos de avaliação, quando comparado ao sistema de pulverização regular a cada sete dias. $\mathrm{O}$ sistema $\mathrm{SE}=0,25$ apresentou o mesmo número de pulverizações do sistema de pulverizações regulares a cada 5 dias, exceto em 2019, que teve uma a menos (Tabela 1).

No sistema $\mathrm{SE}=30$ houve uma redução de 40,33 e 25\% no número de pulverizações, quando comparado ao sistema de aplicação regular semanal, e de 50,60 e $66 \%$, respectivamente quando comparado ao sistema regular a cada cinco dias nos respectivos anos (Tabela 1).

$\mathrm{O}$ sistema de previsão $\mathrm{SE}=20$ resultou em uma pulverização a mais que o sistema $\mathrm{SE}=25$ nos anos de 2017 e 2018. O sistema $\mathrm{SE}=25$ resultou em duas pulverizações a mais que o sistema $\mathrm{SE}=30$ nos três anos de avaliação, predizendo que em condição de menor ocorrência da doença (Figura 1), o número de pulverizações pouco oscilou.

O sistema Botcast (19), utilizado como ferramenta de previsão da queima das pontas das folhas na cultura da cebola avaliado em diversos locais da província de Ontário no Canadá, resultou em redução no número de pulverizações similar ao observado nesse trabalho, com $25 \%$ de aplicação de fungicidas no limite de 21-30 valores de severidade acumulados do modelo proposto para a doença. Resultado semelhante desse trabalho em 2018 foi encontrado com o sistema Blight-alert no estado de Nova York, nos Estados Unidos da América, com redução de $33 \%$ no número de pulverizações (24).

Analisando-se a AACPD, nota-se que os sistemas de previsão utilizando a severidade estimada não diferiram em 2017 dos dois esquemas de pulverizações regulares a cada 5 e 7 dias, e não foram 
Tabela 1. Número de pulverizações, área abaixo da curva de progresso da doença (AACPD) e biomassa fresca (g/planta) de mudas de cebola com queima das pontas das folhas (Botrytis squamosa) tratadas com fungicida conforme três sistemas de previsão ou esquema de controle convencional com pulverizações regulares, a cada cinco ou sete dias

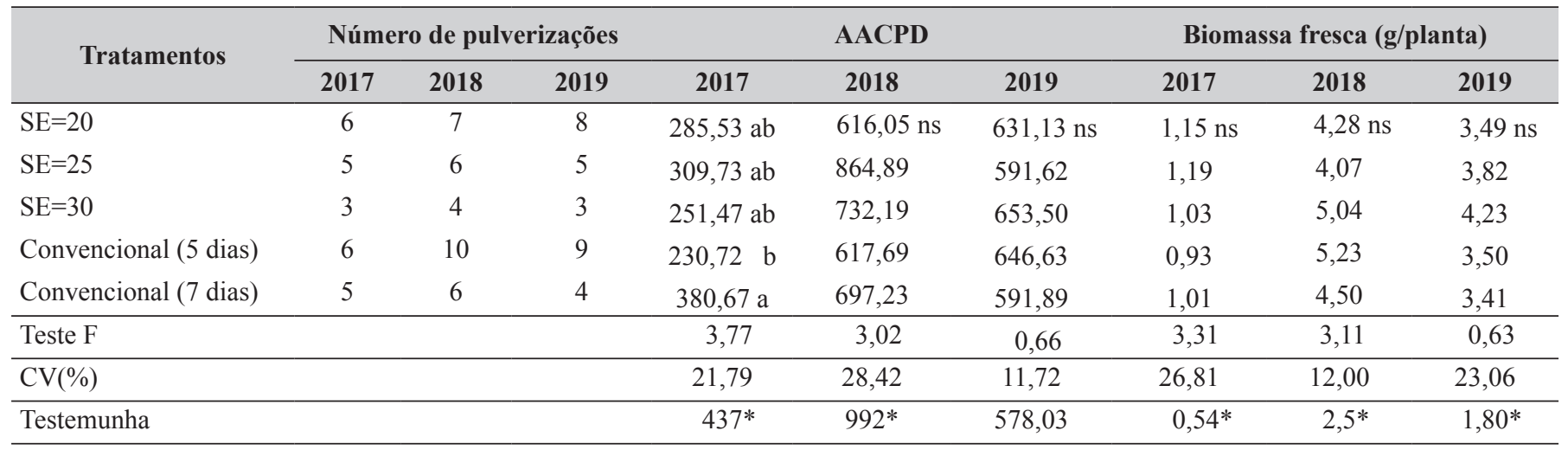

Médias seguidas de mesma letra na coluna não diferem estatisticamente pelo teste de Tukey a nível de 5\%; ns: não significativo pelo teste F; SE: Severidade estimada (\% de severidade/100); CV(\%): Coeficiente de variação. *Média da testemunha difere dos tratamentos por Dunnett 5\%.

significativos entre si em 2018 e 2019, mas diferiram da testemunha em todos os anos de avaliação, evidenciando que a redução do número de pulverizações em relação ao sistema convencional (Tabela 2) é possível devido o menor acúmulo de AACPD durante o ciclo produtivo. Os valores de AACPD ficaram acima dos encontrados por Boff et al. (6), quando avaliaram diferentes preparados em comparação com fungicidas aplicados semanalmente para controle da queima das pontas das folhas na mesma região de estudo desse trabalho.

Apesar de não haver diferença entre os diferentes tratamentos na comparação da $\mathrm{AACPD}$, o sistema de previsão com $\mathrm{SE}=30$ resultou em uma redução de até $33 \%$ dessa variável em relação ao esquema de pulverizações fixas realizadas a cada sete dias, e pouca diferença (máximo de 114,5) da AACPD entre a pulverização a cada cinco dias resultou num acréscimo de 6 pulverizações (Tabela 1), demonstra que nem sempre um número maior de pulverizações reduz a doença, já que o momento correto da aplicação reflete no acumulado da doença ao longo do ciclo da cultura.

Os tratamentos não diferiram nos índices de severidade final (Tabela 2), que oscilou de 26,15 a $34,80 \% ; 27,42$ a $37,61 \%$ e de 45,19 a $53,95 \%$, em 2017, 2018 e 2019, respectivamente. Na testemunha, a severidade final atingiu 41,75; 42,27 e 64,46\%, nos respectivos anos, valores esses acima dos $10 \%$ encontrado por Araújo et al. (1), avaliando a severidade final em plantas pulverizadas a cada dez dias com diferentes fungicidas, e dos $20,2 \%$ quando a pulverização foi semanal (6).

No ciclo de cultivo da cebola, nas safras de 2017 e 2018, a severidade final da doença no sistema convencional de controle com pulverização a cada 5 e 7 dias com o SE=30 oscilou no máximo $10 \%$ de severidade, no entanto em 2017, o $\mathrm{SE}=30$ apresentou $27,2 \%$, enquanto que a cada sete dias, $34,8 \%$, uma diferença de quase $22 \%$ entre eles, porém os valores a cada 5 dias tiveram diferença de apenas 0,9\% (Figura 1). Essa diferença no percentual da doença em 2017 deve se as condições climáticas favoráveis a doença, onde houve um predomínio de alta intensidade pluviométrica $(485 \mathrm{~mm})$ e a doença tem uma correlação significativa com essa variável $(7,24,26)$. No entanto, a taxa de infecção aparente não foi significativa quando se comparou os sistemas convencionais com os sistemas de previsão, mesmo apresentando essa diferença entre os ciclos de avaliação, mas diferindo em todos os anos da testemunha (Tabela 2).

As curvas de progresso da doença nos sistemas de previsão e convencional apresentaram valores de severidade próximos ao longo do tempo em toda a fase de muda da cultura nos três anos de avaliação (Figura 1), no entanto as epidemias diferiram entre os anos, pois a doença iniciou-se a partir dos 42, 35 e 42 dias após a semeadura em 2017, 2018 e 2019, respectivamente (Figura 1). Após seu inicio, as

Tabela 2. Severidade foliar final (\%) e taxa de infecção aparente ( $r$ ) da queima das pontas das folhas da cebola (Botrytis squamosa), após tratamento químico realizado segundo três sistemas de previsão ou controle convencional com pulverizações regulares, a cada cinco ou sete dias

\begin{tabular}{|c|c|c|c|c|c|c|}
\hline \multirow{2}{*}{ Tratamento } & \multicolumn{3}{|c|}{ Severidade foliar final $(\%)$} & \multicolumn{3}{|c|}{$\mathbf{r}$} \\
\hline & 2017 & 2018 & 2019 & 2017 & 2018 & 2019 \\
\hline $\mathrm{SE}=20$ & $30,34 \mathrm{~ns}$ & $31,14 \mathrm{~ns}$ & $48,78 \mathrm{~ns}$ & $0,10 \mathrm{~ns}$ & $0,11 \mathrm{~ns}$ & $0,11 \mathrm{~ns}$ \\
\hline $\mathrm{SE}=30$ & 27,02 & 37,56 & 52,38 & 0,09 & 0,15 & 0,11 \\
\hline Convencional (5 dias) & 26,13 & 27,42 & 53,95 & 0,11 & 0,10 & 0,12 \\
\hline $\mathrm{CV}(\%)$ & 14,84 & 22,06 & 11,44 & 13,27 & 29,33 & 13,56 \\
\hline Testemunha & $41,75^{*}$ & $42,27^{*}$ & $64,46^{*}$ & $0,15^{*}$ & $0,18^{*}$ & $0,16^{*}$ \\
\hline
\end{tabular}

ns: não significativo pelo teste F; CV(\%): Coeficiente de variação; SE: Severidade estimada (\% de severidade/100); *Média da testemunha difere dos tratamentos por Dunnett $5 \%$. 

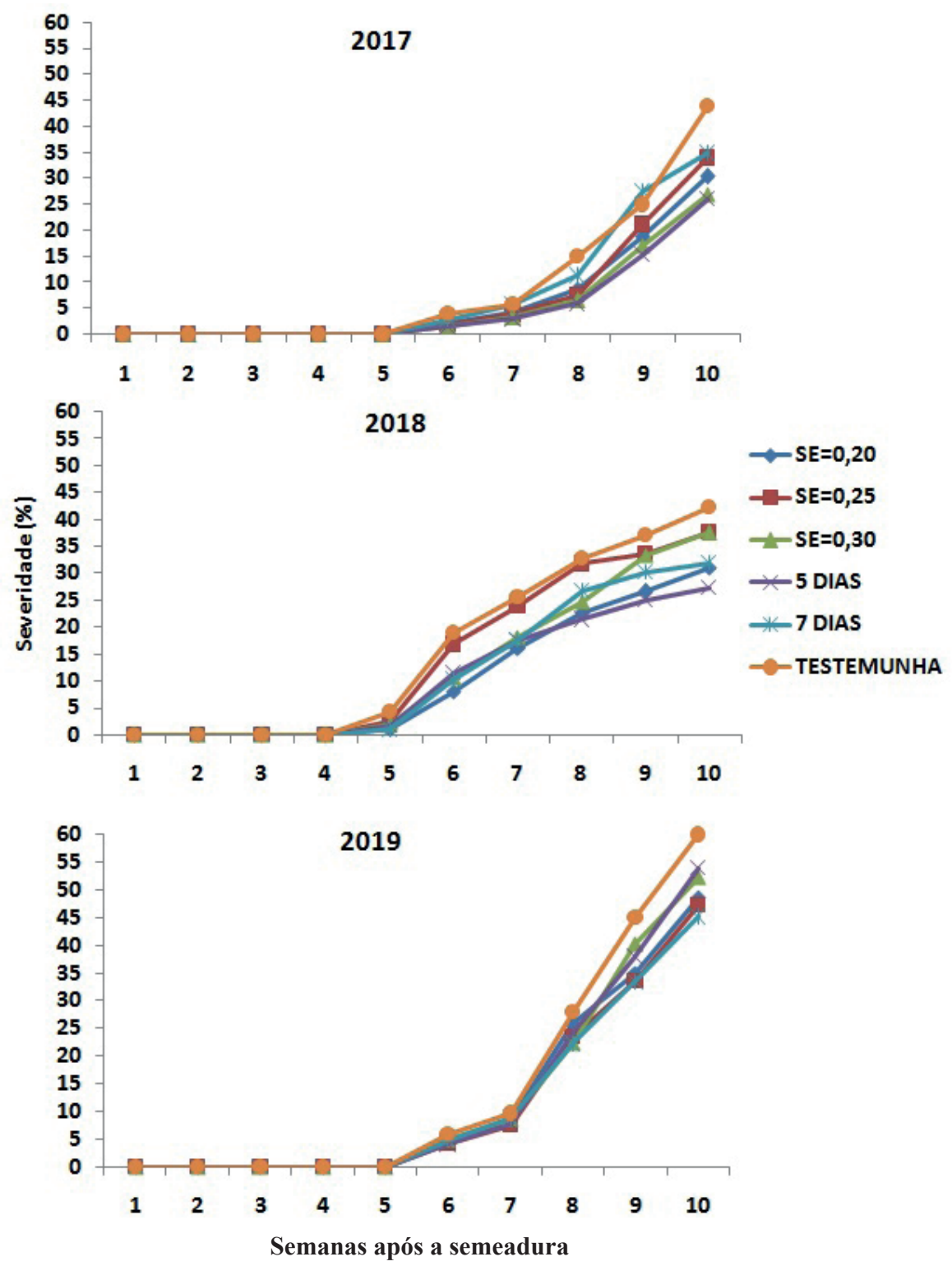

Figura 1. Curvas de progresso da severidade (\%) da queima das pontas das folhas da cebola (Botrytis squamosa) resultantes de três sistemas de previsão ou controle convencional com pulverizações regulares nos ciclos de cultivo de 2017, 2018 e 2019.

epidemias progrediram drasticamente em curto período de tempo $(5$ a 6 semanas) (Figura 1), efeito também constatado por Sutton et al. (20). Apesar das curvas ficarem muitos próximas entre os sistemas, foi constatada diferença entre a testemunha nos três ciclos. A diferença da maior severidade final entre os sistemas de previsão e a testemunha foi de $14,73 \%$ em 2017 , e 4,71 e 12,08\% em 2018 e 2019, respectivamente (Tabela 2).

Os tratamentos não diferiram entre si para a biomassa fresca das mudas nos três ciclos de avaliação (Tabela 1). A biomassa no $\mathrm{SE}=30$ foi 47, 50 e 57\% superior a testemunha em 2017, 2018 e 2019, respectivamente, evidenciando a sua drástica redução pela doença. Nesse trabalho constatou-se que a biomassa pouco oscilou entre os tratamentos aplicados: sistemas de previsão e convencionais, mas redução significativa do número de pulverizações foi constatada quando se compara o sistema de previsão que utiliza $\mathrm{SE}=30 \mathrm{com}$ o sistema convencional com pulverizações regulares a cada 7 dias, mas sem comprometer o rendimento de muda. O menor incremento foi verificado em 2019 (Tabela 1), apesar da doença, deve-se ainda a intensa precipitação pluvial concentrada e dias nublados que ocorreu durante a fase final da produção de mudas.

Segundo Teng (21), o rigor da avaliação não deve ser exagerado, uma vez que o sistema é uma aproximação da realidade e durante o processo de validação, se necessário, pode se ter um espaço entre o que é estimado e o real, para aprimoramento contínuo do sistema (8). O sistema proposto permitiu demonstrar que pode ser muito útil como ferramenta do manejo da queima das pontas, já que a validação do sistema tem por objetivo determinar se o comportamento do sistema real é coerente com o modelo $(3,4,11)$.

A queima das pontas das folhas é uma das principais preocupações fitossanitárias dos produtores de cebola do Alto Vale do Itajaí e regiões com microclima semelhante. Considerando o aspecto econômico para o produtor, a utilização do sistema de previsão com $\mathrm{SE}=30$ leva a uma 
redução no custo operacional, em decorrência da diminuição de até $40 \%$ no número de pulverizações e, consequentemente, menor exposição dos aplicadores de agrotóxico sem perda da produtividade. Quanto ao meio ambiente, pode haver uma redução significativa do impacto que o agrotóxico causa no ecossistema.

O uso do sistema de previsão mostrou ser uma ferramenta viável no manejo da queima das pontas das folhas da cebola em Santa Catarina e encontra-se disponível gratuitamente para uso da cadeia produtiva na plataforma Agroconnect da EPAGRI-CIRAM disponível em http:// www.ciram.sc.gov.br/agroconnect/ no menu atividade agropecuária cebola, alerta fitossanitário.

\section{AGRADECIMENTOS}

O autor agradece o apoio financeiro recebido do Conselho Nacional de Desenvolvimento Científico e Tecnológico - CNPq pela bolsa de iniciação científica PIBITI/Cnpq de Bruna Kotkoski, IFC/Campus Rio do Sul de Cristiane Wernke e ao técnico de campo, Marcio Rampelotti pela execução das pulverizações.

\section{REFERÊNCIAS}

1. Araújo, E.R.; Alves, D.P.; Higashikawa, F.S. Cultivar resistence and chemical, biological and fertilizer treatments for controlling Botrytis leaf blight of onion. Tropical plant pathology, Brasília, DF, v.43, n.2, p.160-164, 2018.

2. Barreto, M.; Vale, F.X.R.; Paul, P.A.; Scaloppi, E.A.G.; Andrade, D.F.A.A. Sistemas de previsão e estação de aviso. In: Vale, F. X. R.; Jesus Junior, W. C.; Zambolim, L (Ed.). Epidemiologia aplicada ao manejo de doenças de plantas. Belo Horizonte: Perffil, 2004. cap.6, p.243-266.

3. Bergamim Filho, A.; Amorim, L. Doenças de plantas tropicais: epidemiologia e controle econômico. São Paulo: Ceres, 1996. 299p.

4. Bergamim Filho, A.; Amorim, L. Sistemas de previsão e avisos. In: Bergamim Filho, A.; Kimati, H.; Amorim, L. (Ed.). Manual de fitopatologia. 3. ed. São Paulo: Ceres, 1995. v. 1, cap. 31, p.627-646.

5. Boeing, G. Fatores que afetam a qualidade da cebola na agricultura familiar Catarinense. Florianópolis: Instituto CEPA, 2002. 88p.

6. Boff, P.; Gonçalves, P.A.S.; Debarba, J.F. Efeito de preparados caseiros no controle da queima-acizentada, na cultura da cebola. Horticultura brasileira, Brasília, DF, V.17, n.2, p.81-85, 1999.

7. Duffeck, M.R.; Marcuzzo, L.L. Influência da precipitação pluvial sobre a severidade da queima das pontas (Botrytis squamosa) em canteiro de mudas de cebola. In: Dia de Pesquisa, Ensino, Produção e extensão - PEPE, Rio do Sul. Anais. Rio do Sul: IFC, 2011. 1 CD-ROM.

8. Fernandes, J.M.C.; Maffia, L.A. Simulação de epidemias. Revisão Anual de Patologia de Plantas, Passo Fundo, v.2, p.293-334, 1994.

9. IBGE. Cebola, Estatística da produção agrícola, setembro de 2018. IBGE: Brasília, setembro, 2018.
10. Jesus Junior, W.C.; Pozza, E.A.; Vale, F.X.R.; Anguilera, G.M. Análise temporal de epidemias. In: Vale, F. X. R.; Jesus Junior, W. C.; Zambolim, L. (Ed.). Epidemiologia aplicada ao manejo de doenças de plantas. Belo Horizonte: Perffil, 2004. cap.4, p.127-191.

11. Kranz, J.; Hau, B. Systems analysis in epidemiology. Annual Review of Phytopathology, Palo Alto, v.18, n.1, p.67-83, 1980.

12. Krause, R.A.; Massie, L.B. Predictive systems: modern approaches to disease control. Annual Review of Phytopathology, Palo Alto, v.13, n.1, p.31-47, 1975.

13. Marcuzzo, L.L.; Nascimento, A.; Kotkoski, B. Technique for inducing Botrytis squamosa sclerotium formation in vitro. Summa phytopathologica, Botucatu, v.43, n.3, p.251, 2017.

14. Marcuzzo, L.L.; Haveroth, R. Development of a weather-based model for Botrytis leaf blight of onion. Summa phytopathologica, Botucatu, v.42, n.1, p.92-93, 2016.

15. Menezes Júnior, F.O.G; Marcuzzo, L.L. Manual de boas práticas agrícolas: sustentabilidade das lavouras de cebola do estado de Santa Catarina Florianópolis: Epagri, 2016, 143p.

16. Mizubuti, E.S.G. Sistema de previsão de doenças de plantas: uma ferramenta útil? In: Zambolim, L. (Ed). $\mathbf{1}^{\circ}$ Encontro de manejo integrado de doença e pragas. Viçosa: Universidade Federal de Viçosa, 1999. p.42-46.

17. Reis, E.M. Previsão de doenças de plantas. Passo Fundo: Universidade de Passo Fundo, 2004. 316p.

18. Sutton, J.C. Predictive value of weather variables in the epidemiology and management of foliar disease. Fitopatologia Brasileira, Brasília, DF, v.13, n.4, p.305-312, 1988.

19. Sutton, J.C.; James, T.D.W.; Rowell, P.M. Botcast: a forecasting system to time initial fungicide spray for managing botrytis leaf blight of onions. Agriculture, Ecosystems and Environment, Amsterdam, v.18, n.2, p.123-143, 1986.

20. Sutton, J.C.; James, T.D.W.; Rowell, P.M. Relation of weather and host factors to an epidemic of botrytis leaf blight in onions. Canadian Journal of Plant Pathology, Ottawa, v.5, n.4, p.256-265, 1983.

21. Teng, P.S.A. Comparison of simulation approaches to epidemic modeling Annual Review of Phytopathology, Palo Alto, v.23, p.351-379, 1985.

22. Vale, F.X.R.; Zambolim, L. Influência da temperatura e da umidade nas epidemias de doenças de plantas. Revisão Anual de Patologia de Plantas, Passo Fundo, v.4, p.149-207, 1996.

23. Vanderplank, J.E. Plant disease: epidemics ant control. New York: Academic Press, 1963. 349p

24. Vincelli, P.C.; Lorbeer, J.W. BLIGTH-ALERT: A weather-based predictive system for timing fungicide applications on onion before infection periods of Botryts squamosa. Phytopathology, St. Paul, v.79, n.4, p.493-498, 1989.

25. Vincelli, P.C.; Lorbeer, J.W. Forecasting spore episodes of Botrytis squamosa in commercial onion fields in New York. Phytopathology, St. Paul, v.78, n.7, p.966-970, 1988.

26. Vincelli, P.C.; Lorbeer, J.W. Relationship of precipitation probability to infection potencial of Botrytis squamosa on onion. Phytopathology, St Paul, v.78, n.8, p.1078-1082, 1988.

27. Wordell Filho, J. A.; Boff, P. Queima acizentada - Botrytis squamosa Walker. In: Wordell Filho, J.A.; Rowe, E.; Gonçalves, P.A.; Debarba, J.F.; Boff, P.; Thomazelli, L.F. Manejo Fitossanitário na cultura da cebola Florianópolis: EPAGRI, p.19-30, 2006. 